\title{
Tin Qubits Give Diamond a New Shine
}

\author{
Nanophotonic devices based on tin-vacancy qubits in diamond show promise as building \\ blocks of quantum repeaters, an important step toward the realization of long-range quantum \\ networks.
}

\section{by Evangelia Takou ${ }^{1}$ and Sophia E. Economou ${ }^{1}$}

$\mathrm{B}$ uilding long-range quantum networks is one of the most important and ambitious goals of quantum science and engineering. To connect these networks into a quantum internet will require intermediate stations where quantum information-carried by photons - can be manipulated and "refreshed." These stations, known as quantum repeaters, contain long-lived qubits with an optical interface that allows the photons to be entangled with the spins encoding these qubits. Color centers in solids are prime candidates for quantum repeaters, as they can have long coherence times, spin-selective optical transitions, and compatibility with photonic devices, such as cavities, that facilitate photon emission and routing. The

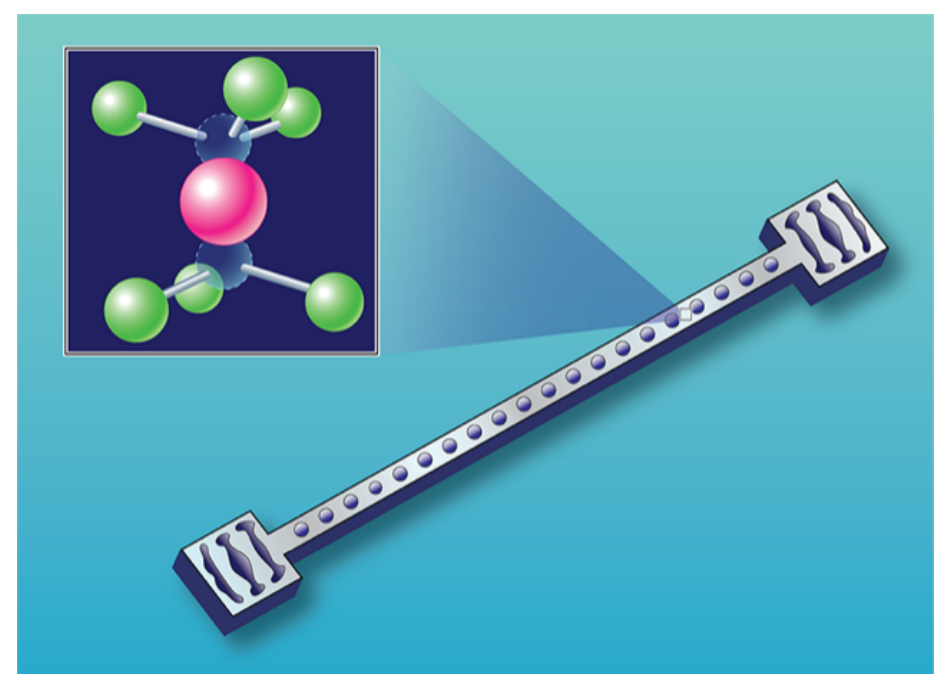

Figure 1: A photonic crystal cavity in diamond. Researchers implanted tin atoms in the crystal, producing tin-vacancy (SnV) centers (one of which is shown in the inset). When the cavity's frequency is tuned to match that of the SnV centers, the photon emission is enhanced by a factor of 40 , demonstrating that these centers could become the building blocks of repeaters in a quantum network. (APS/Carin Cain)

\footnotetext{
${ }^{1}$ Department of Physics, Virginia Tech, Blacksburg, VA, USA
}

tin-vacancy $(\mathrm{SnV})$ center in diamond, a relatively new and promising color center [1], features several of these key elements. Until recently, however, these $\mathrm{SnV}$ centers had not been integrated into cavities in which their emission would be enhanced. Now, a team led by Jelena Vučković at Stanford University has succeeded in integrating an SnV center into a nanophotonic device and has achieved $90 \%$ photon emission into the desired cavity mode [2]. This work is an important step toward the realization of long-range quantum networks.

A color center forms in a crystalline solid when one or more lattice atoms are missing or substituted by another species. Such complexes can absorb and emit light and, if they have a ground state with nonzero spin, can be used as a qubit. Intense research is dedicated to exploring color centers in diamond as building blocks of quantum networks [3]. The most studied diamond color center for quantum technology applications is the nitrogen vacancy (NV). With its remarkably long coherence time and spinselective transition, the NV center has played a central role in the development of quantum networks, including the recent seminal demonstration of a three-node network with entanglement swapping capabilities [4]. Despite its successes, though, the NV suffers from a few shortcomings that limit its suitability for quantum networks. One issue is vibrational noise, which causes the majority of photons to be emitted incoherently, thereby reducing the success probability of spin-photon entanglement schemes. The other issue is the permanent electric dipole of this color center, leading to sensitivity to nearby charges. Such charge noise, which can destroy information stored in a qubit, is exacerbated when the NV is near surfaces, as in nanophotonic devices.

These limitations have led researchers to consider alternative qubits. A prominent family of diamond color centers currently under intense investigation consists of complexes made up of two carbon vacancies between which is a groupIV atom, an atom with four valence electrons. The inversion symmetry of these systems leads to a vanishing permanent electric dipole moment, making them suitable for integration in nanophotonic structures. Moreover, most of the emitted photons have frequencies in the desirable "zerophonon line," where there is no vibrational noise. One of the most studied group-IV centers in diamond is the silicon-vacancy $(\mathrm{SiV})$ center, which has been integrated into 
nanophotonic devices and used in the first demonstration of memory-enhanced quantum communication [5]. However, the operation of an $\mathrm{SiV}$ center requires complex and expensive cryogenic technology based on dilution refrigeration to reach temperatures at which the coherence times are sufficiently long for applications [6].

The SnV center [7] has emerged as a potential solution. It has a number of desirable attributes: It obeys inversion symmetry, making it insensitive to charge noise; it emits photons primarily through the zero-phonon line; it exhibits longer coherence times than $\mathrm{SiV}$; and it can be operated at a few kelvin, a temperature that can be reached with simpler technology than dilution refrigerators. These features make $\mathrm{SnV}$ particularly promising for use as a quantum network node. While optical initialization of $\mathrm{SnV}$ spins has been demonstrated [7], this color center had not-until now-been integrated into nanophotonic structures, as required for applications.

For their demonstration, the Stanford team fabricated high-quality nanophotonic cavities from a diamond plate [2]. They first implanted tin atoms within the diamond. Typically, this integration of heavy impurity atoms into nanophotonic devices comes at the price of damaging the diamond surface and degrading the emitter quality. The authors overcame this problem using their novel color-center generation method that ensures precise placement of Sn impurity atoms below a high-quality diamond substrate [8]. The team then constructed photonic crystal cavities into the diamond plate. Each long, thin cavity had an array of holes etched along it. The cavities were also partly suspended in the air to make the light confinement stronger.

The researchers succeeded in efficiently coupling light in and out of the cavities via "inverse-designed" couplers that they developed in earlier work [9]. They could tune the cavity wavelength by depositing condensed argon on the device. When the wavelength of the cavity matched that of the optical transition of the color centers, the team observed a 40-fold enhancement of emission intensity compared with the off-resonant case. Furthermore, while the photons retained their sharp linewidths, the spontaneous emission rate was considerably enhanced. This narrow emission meant that photons have nearly $100 \%$ probability of being emitted into a single cavity mode. These elements are necessary for establishing high enough entanglement rates in quantum repeaters.

These new results, together with the recent demonstration of coherent optical control of the SnV spin state [10], are key steps toward the creation of SnV-based networks. The remaining critical step toward this goal is the creation of a high-fidelity quantum memory register composed of carbon-13 atoms - the only isotope of carbon with nonzero spin-in the diamond lattice that can store quantum information transferred to and from the $\mathrm{SnV}$ center. Upon such a demonstration, the $\mathrm{SnV}$ center would be in a position to outshine its diamond-based predecessors.

\section{REFERENCES}

[1] T. Iwasaki et al., "Tin-vacancy quantum emitters in diamond," Phys. Rev. Lett. 119, 253601 (2017).

[2] A. E. Rugar et al., "Quantum photonic interface for tin-vacancy centers in diamond," Phys. Rev. X 11, 031021 (2021).

[3] M. Ruf et al., arXiv:2105.04341.

[4] M. Pompili et al., "Realization of a multinode quantum network of remote solid-state qubits," Science 372, 259 (2021).

[5] M. K. Bhaskar et al., "Experimental demonstration of memoryenhanced quantum communication," Nature 580, 60 (2020).

[6] D. D. Sukachev et al., "Silicon-vacancy spin qubit in diamond: A quantum memory exceeding $10 \mathrm{~ms}$ with single-shot state readout," Phys. Rev. Lett. 119, 223602 (2017).

[7] M. E. Trusheim et al., "Transform-limited photons from a coherent tin-vacancy spin in diamond," Phys. Rev. Lett. 124, 023602 (2020).

[8] A. Rugar et al., "Generation of tin-vacancy centers in diamond via shallow ion implantation and subsequent diamond overgrowth," Nano Lett. 20, 1614 (2020).

[9] C. Dory et al., "Inverse-designed diamond photonics," Nat. Commun. 10, 3309 (2019).

[10] R. Debroux et al., "Quantum control of the tin-vacancy spin qubit in diamond," arXiv:2106.00723.

10.1103/Physics.14.105 